\title{
Rapid communication \\ The accuracy of fluoride measurement in water and its implications for water fluoridation
}

\author{
Johannes Haarhoff \\ Rand Afrikaans University, PO Box 524, Auckland Park 2006, South Africa
}

\begin{abstract}
The accurate measurement of the fluoride concentration in water is an essential prerequisite to stay within the allowable dosing tolerances required by the South African water fluoridation legislation. In the absence of reliable error estimates for fluoride measurement in natural water samples, a study was conducted utilising data from interlaboratory comparison studies conducted by the CSIR, the SABS and MEDUNSA. This study shows that:

- natural samples are more difficult to measure than synthetic samples;

- technology advances over the last 25 years did not reduce the measurement error significantly;

- all analytical methods suffer to some extent when natural samples are analysed; and

- the measurement error will have to be appreciably reduced if the legal requirements of the pending water fluoridation are to be met.
\end{abstract}

Keywords: Water fluoridation, fluoride measurement, accuracy, measurement error, natural samples, synthetic samples

\section{Introduction}

South Africa is poised for the mandatory fluoridation of drinking water up to a general target concentration of $0.7 \mathrm{mg} \mathrm{F} / \mathrm{l}$. This often emotional issue had been debated during years of preparation of draft legislation, until the approval of the final regulations by Parliament and their promulgation in September 2001. The attention of the water industry has therefore moved to the many smaller but important technical issues of practical implementation. One of the obvious keystones of water fluoridation is the ability to measure the fluoride concentration in water - firstly for determining how much supplementation is required, and secondly for checking that the target is reached within the allowable tolerances. This paper thus directs its attention to the measurement of fluoride in water; a vital, difficult aspect of water fluoridation which has seemingly been overlooked.

\section{Dosing accuracy legally required in SA}

The SA Regulations (Government Gazette, 2001) are explicit in their requirements for dosing accuracy, thereby closely following similar UK regulations. The first stipulation is:

- Instantaneously measured fluoride concentration must be within $0.2 \mathrm{mg} / \mathrm{l}$ of the target.

The fluoridation plant operator therefore has to measure the fluoride concentration in the raw water; measure the water flow rate; calculate the fluoride dosing rate to make up the shortfall towards the target concentration; and check the procedure by measuring the fluoride concentration in the fluoridated water. For the purposes of

III. +2711489 2148; fax: +27 11489 2148; e-mail: jh@ing.rau.ac.za Received 3 March 2003. this paper, assume that there are no calculation errors, that the water flow rate and the fluoride solution strength in the day tank are exactly known, and that the fluoride dosing pump can be set with absolute accuracy. The only error to consider is therefore the error due to the measurement of the fluoride concentration before and after dosing.

It is obvious that any measurement error will be compounded during the required feedback loop. This is illustrated by the following example, where a constant under-measurement error of $30 \%$ is assumed for illustration:

- The raw water fluoride concentration is actually $0.20 \mathrm{mg} / \mathrm{l}$, but measured as $0.14 \mathrm{mg} / \mathrm{l}$. The shortfall is therefore actually 0.50 $\mathrm{mg} / \mathrm{l}$ below the target level of $0.70 \mathrm{mg} / \mathrm{l}$, but perceived by the operator to be $0.56 \mathrm{mg} / \mathrm{l}$.

- The dosing pump is set to add $0.56 \mathrm{mg} / \mathrm{l}$, resulting in an actual concentration of $0.76 \mathrm{mg} / \mathrm{l}$ or $0.06 \mathrm{mg} / \mathrm{l}$ above the target. The operator measures this as $0.53 \mathrm{mg} / \mathrm{l}$ and still perceives a shortfall of $0.17 \mathrm{mg} / \mathrm{l}$.

- The operator, correctly, increases the dosing rate by $0.17 \mathrm{mg} / \mathrm{l}$ to get an actual concentration of $0.93 \mathrm{mg} / \mathrm{l}$, which is still perceived as $0.65 \mathrm{mg} / \mathrm{l}$ or $0.05 \mathrm{mg} / \mathrm{l}$ short of the target.

- This cycle continues until the concentration stabilises at the perceived target concentration. By this time, the actual concentration is about $1.00 \mathrm{mg} / \mathrm{l}$, or about a $40 \%$ overdose.

As an apparent independent check on the dosing accuracy, the SA Regulations further stipulate that:

- Average dosage over a week must be within $0.1 \mathrm{mg} / \mathrm{l}$ of the target.

The fluoridation operator should check this requirement by a careful site inventory of on-site fluoridation chemicals and the volume of water fluoridated since the previous check. (It should 


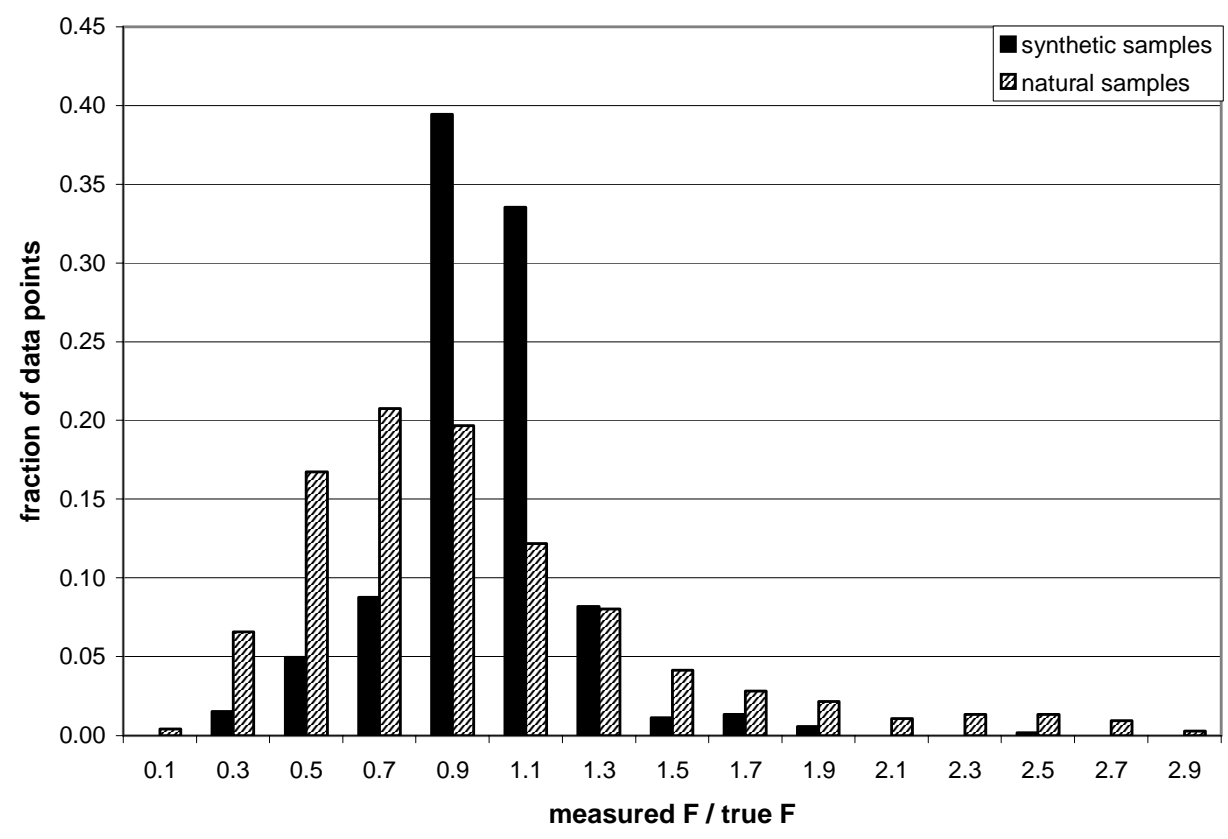

Figure 1

Histogram of data points for synthetic and natural samples respectively

certainly not be checked by mathematical averaging of the spot measurements taken during the week, as this would not detect an error such as demonstrated above!). An inventory check will provide an external check on how much fluoride was dosed. To then obtain the average concentration in the fluoridated water, the fluoride dosed has to be added to the concentration originally present, which can be obtained in no other way than taking the volume-weighted average of the raw water concentrations measured during the week. It is clear that an inventory check is still dependent on the accuracy of measuring the fluoride concentration in the raw water. Using the same example as above where the raw water concentration is undermeasured by $0.60 \mathrm{mg} / \mathrm{l}$, the target will therefore also be missed by $0.60 \mathrm{mg} / \mathrm{l}$ without knowing.

This line of reasoning underscores the vital importance of measuring the fluoride concentration accurately. For the proper estimation of risk, it is essential to get a quantitative grip on the practical limits of the error of fluoride measurement. Despite numerous enquiries by the author, no comprehensive or convincing information could be readily obtained. As the author is neither an analytical chemist nor a statistician, it is hoped that this cursory analysis will stimulate further, more comprehensive consideration of this question by others.

\section{Data sources and exclusion criteria}

The Division of Water Technology (DWT) of the CSIR ran an interlaboratory comparison programme from the middle of the 1970s to 1998. A wide variety of sample types and analytical methods were covered for water, sewage, sediments and sludges. As part of this programme, samples for fluoride analysis were distributed on eight occasions, comprising both synthetic samples (made up of distilled water with reagent-grade chemicals) and natural samples (surface water, effluent and groundwater). In total, 21 samples were sent out between 1977 and 1998, with an average of 35 laboratories participating in each survey. A total of 725 data points were collected. The DWT followed a consistent approach of removing all the outliers with a statistical procedure before reporting. For the study reported here, all the original data points, outliers included, were retrieved from old files and recaptured electronically.
The South African Bureau of Standards (SABS) Test House runs an ongoing WATERCHECK programme which covers more than 70 analytical laboratories in Southern Africa. Fluoride is part of a group of determinants which is measured every third month on three samples (one synthetic and two natural samples). The latest annual data set for 2002 was retrieved, during which a total of 631 fluoride values were returned from 55 laboratories. No outliers are removed in the reporting process adopted by the SABS, but a socalled robust statistical evaluation is used to reduce the effect of outliers. For this study, all the original values, outliers included, were recaptured electronically.

A small once-off survey was conducted by the Medical University of Southern Africa (MEDUNSA) during 2002. Five samples of tap water, spiked to varying degrees with fluoride compounds, were sent to 10 commercial laboratories in the Gauteng area for fluoride analysis, with a clear note that it formed part of an interlaboratory comparison. This data set, in its entirety, was included in this study.

In an operational environment, the operator has to react to each measurement as it is taken. Outliers, on the other hand, can only be detected and rejected after some time, when a retrospective statistical analysis becomes possible. For this reason, all data points were critically considered for this analysis. Six complete sample sets were excluded on the basis of:

- Two samples were spiked to more than $20 \mathrm{mg} / \mathrm{l}$, which is way outside the range where water fluoridation plants will ever operate.

- One laboratory returned values for a set of four samples where every single value was deemed to be an outlier by the DWT procedure, which indicated a gross, systematic error which warranted exclusion from the data set.

A number of individual data points were removed next, as they were obviously extreme outliers:

- Individual measurements which were less than $20 \%$ of the average concentration were excluded. This eliminated 12 of the data points.

- Individual measurements which were more than five times the 
Figure 2

Cumulative distribution plot of fluoride measurement error, showing difference between data sets and sample types

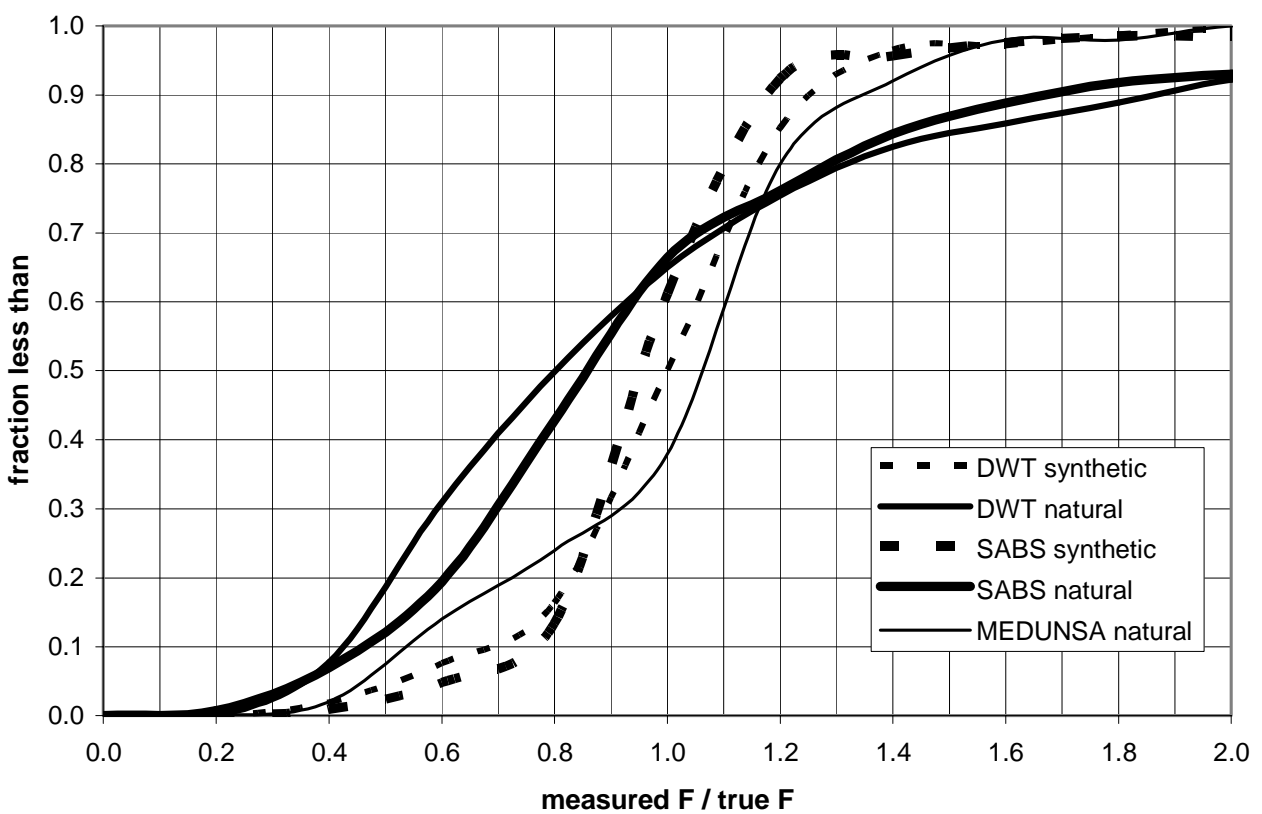

average concentration were excluded. This eliminated 31 of the data points.

This left a total of 1278 data points, including 36 samples over 25 years, covering a range of average concentrations between $0.07 \mathrm{mg} / \mathrm{l}$ and $4.37 \mathrm{mg} / \mathrm{l}$. Eighty per cent of the samples had average concentrations of between $0.2 \mathrm{mg} / \mathrm{l}$ and $2.1 \mathrm{mg} / \mathrm{l}$.

In synthetic samples, the "true" fluoride concentration is known from the way the samples are made up. In some cases, they were reported, but they were not used for this study. For the purposes of this study, the "true" fluoride concentration of each sample was taken as the arithmetic mean of all the measurements reported for that sample, after removal of the outliers as described above. All the data points were then finally normalised by dividing each value by the "true" value for that sample.

\section{Data analysis}

\section{Natural vs. synthetic samples}

As with most analytical procedures, care has to be taken to eliminate interferences by background chemical compounds. Fluoride, to name but one example, will form complexes with aluminium which will affect both aluminium and fluoride measurements if no precautions such as preliminary distillation or digestion are taken. During the DWT programme, the consistency of fluoride measurements generally suffered from a high percentage of outliers attributed to not adequately pretreating the samples.

In natural samples, as opposed to synthetic samples, there are many more unknowns that could affect the fluoride measurements. The hypothesis then has to be that accuracy of fluoride measurements in natural samples has to be poorer than in synthetic samples. The data set was therefore split into a "natural" group (747 data points from 21 samples) and a "synthetic" group (525 data points from 15 samples). Figure 1 shows the distribution of the data in histogram form. Two points emerge from Fig. 1:

- The synthetic samples are more narrowly distributed, as hypothesised above. The remainder of this analysis will thus be carried through by keeping natural and synthetic data separate.
- There is a definite asymmetry in the distribution, especially evident for the natural samples. This is due to the fact that the distribution is constrained on the left by a zero concentration limit. The normal distribution is therefore not adequate for describing fluoridation accuracy; the log-normal distribution would be a more suitable candidate should someone wish to pursue the modelling of the measurement error.

\section{"Old" vs. "new" technology}

The DWT data set spans the period 1977 to 1998, therefore including data collected by what many would consider to be "old" technology. The SABS and MEDUNSA data sets were collected during 2002 and are a reflection of the current state of technology used in South African laboratories. An obvious question is whether the much-touted advances in instrument technology led to a significant, if any reduction in measurement error.

The data set was next split by both sample type (natural and synthetic) and data set (DWT, SABS and MEDUNSA), yielding the following number of samples per subset:
- DWT synthetic

- DWT natural

- SABS synthetic

- SABS natural

- MEDUNSA natural

\section{4 data points from 11 samples 297 data points from 8 samples 209 data points from 4 samples 402 data points from 8 samples 50 data points from 5 samples}

These data were used to construct cumulative distribution lines, which are shown in Fig. 2. The following points emerge from Fig. 2:

- The difference between natural and synthetic samples observed earlier, remains strongly evident for the individual data sets.

- There is very little, if any, difference between the "old" data set of the DWT and the "new" data set of the SABS. This is evident from a comparison of both the synthetic and natural sample lines. Advances in instrument technology have made no appreciable difference to the empirically observed measurement accuracy. 


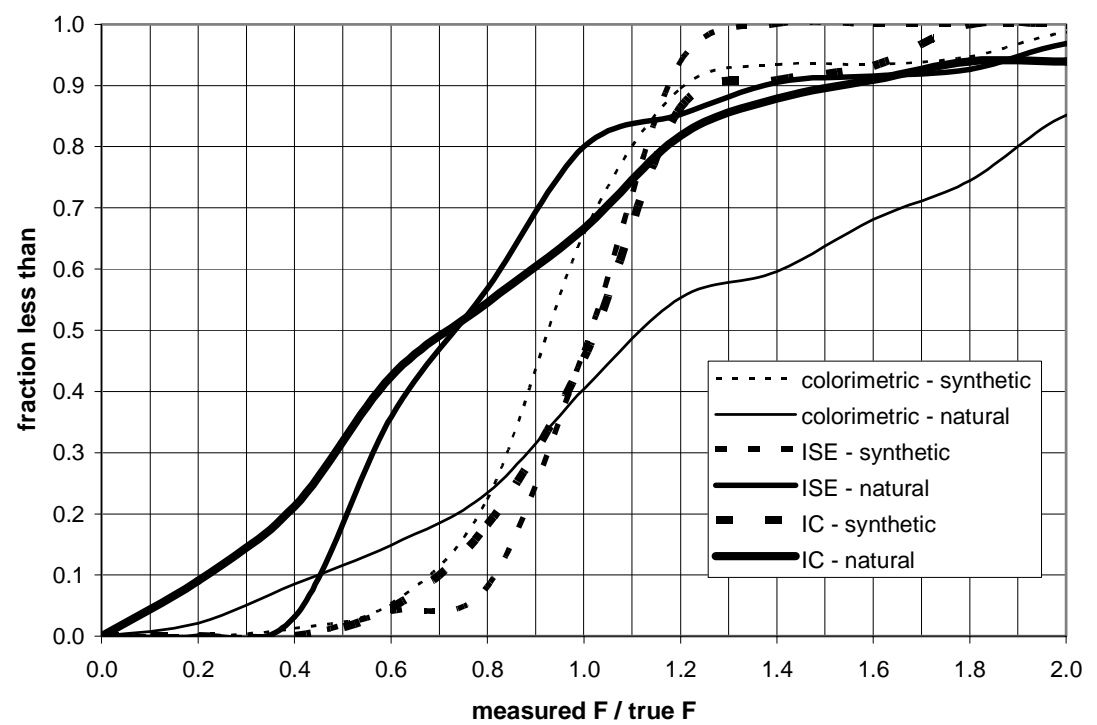

Figure 3

Cumulative distribution plot of fluoride measurement error, showing difference between analytical methods and sample types

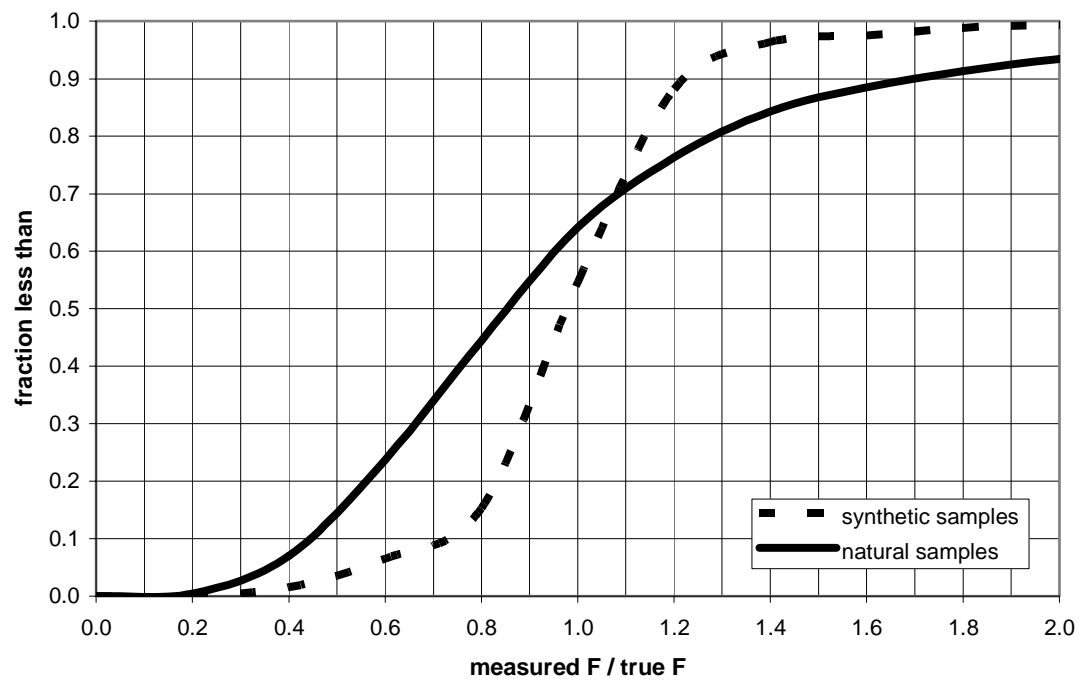

Figure 4

Cumulative distribution plot of fluoride measurement error, showing difference between natural and synthetic samples

- The MEDUNSA data set does not clearly follow either the natural or the synthetic data. This is understood if the nature of the MEDUNSA samples is explained. One sample was a tap water sample with an average concentration of $0.07 \mathrm{mg} / \mathrm{l}$. The other four samples of the set were made up of the same tap water, progressively spiked to concentrations of $0.19 \mathrm{mg} / \mathrm{l}$, $0.45 \mathrm{mg} / \mathrm{l}, 0.87 \mathrm{mg} / \mathrm{l}$ and $1.18 \mathrm{mg} / \mathrm{l}$ respectively. The samples themselves could therefore be considered to be progressively more "synthetic" than natural.

\section{Comparison of analytical methods}

Fluoride in water can be measured in a number of ways. There are colorimetric methods (of which the SPADNS method is the most commonly used), the ion-selective electrode (ISE) method, and ion chromatography (IC), all comprehensively described in Standard Methods (1985). For routine measurement on site, the practical choice is between SPADNS (only an option when spot analysis is opted for rather than continuous monitoring) or ISE (suitable for both spot and continuous measurement). During the course of the DWT study, it seemed that the SPADNS method was more susceptible to these interferences and it was suggested to SPADNS users that they should consider switching to the ISE technique.

The SABS and MEDUNSA data sets do not have information on the methods used by the participating laboratories, but the DWT data set included such information between 1977 and 1990, which includes $35 \%$ of all the data considered in this study. The methodspecific data points break down to:

- Natural samples measured colorimetrically 47 data points

- Synthetic samples measured colorimetrically 76 data points

- Natural samples measured by ISE 95 data points

- Synthetic samples measured by ISE 148 data points

- Natural samples measured by IC 33 data points

- Synthetic samples measured by IC 44 data points

The cumulative distribution plots for these six groups are shown in Fig. 3. The following points emerge from Fig. 3:

- For synthetic samples, there is little difference amongst the three methods. All follow the same narrow distribution seen in the earlier analyses.

- For natural samples, all the methods show significantly poorer accuracy than for synthetic samples. The effect of interfering compounds cannot be eliminated by simply switching the analytical method.

- The colorimetric method returns higher concentration values than the ISE and IC methods.

- The ISE and IC methods return comparable results for natural samples. 


\section{Likely fluoride measurement accuracy}

Analytical laboratories commonly use the "standard deviation approach" as a scoring criterium during interlaboratory comparison studies. Both the DWT and the SABS programmes consider results within 1 standard deviation from the mean as "good", between 1 and 2 deviations away as "average" and further than 2 standard deviations as "poor". For normal distributions (normally implicitly or explicitly assumed), this translates into a criterium that $68 \%$ of all measurements would be "good" and a further $27 \%$ of all measurements would be "average". A more conservative, arbitrary assumption of only $50 \%$ "good" values is adopted for the purposes of this paper - an assumption of the author.

Figure 4 shows the cumulative distribution plot of the entire pooled data set, split between natural and synthetic samples. The $25^{\text {th }}$ and $75^{\text {th }}$ percentile lines from Fig. 4 provide error estimates of the measurement error that could reasonably be expected from "good" laboratories. These values are shown in Table 1.

\section{Questions pertaining to pending water fluoridation}

Does the performance of analytical laboratories give a reliable indication of measurement performance at an operational water fluoridation plant?

This is a crucial question which cannot yet be answered. Intuitively, there are reasons why analytical laboratories should do better than operational plants:

- Recognised analytical laboratories that care to participate in nation-wide comparisons invariably have trained chemists working under professional supervision and clean, controlled laboratory conditions.

- It is only human that each participating laboratory will put its best possible foot forward at the time of the comparative analyses. It is difficult if not impossible to sustain the same diligence over the many years of continuous operation of a water fluoridation plant.

Conversely, it can be argued that operational plants should do better:

- The interlaboratory comparisons normally request a suite of analyses of which fluoride forms only a small part. A control laboratory dedicated to a single compound may be better focused and equipped to produce better results.

- Many of the participating laboratories do not specialise in water analyses, but probably in some other areas, for example air, food, coal, soils, etc.

\section{Were the samples of this study representative of general water treatment conditions?}

Those samples with unrealistically high concentrations were excluded from the analysis. The data from synthetic samples were analysed separately from the data from natural samples. A small number of borehole and sewage effluent samples remained in the data set, but in practice these sources could also be encountered. In general, the synthetic samples were spiked to F concentrations above $1 \mathrm{mg} / \mathrm{l}$, while most natural samples had concentrations less than $1 \mathrm{mg} / \mathrm{l}$. The very low concentrations of say less than 0.3 $\mathrm{mg} / \mathrm{l}$ were poorly represented in the data set and it seemed from the
TABLE 1

Fluoride measurement limits based on $50 \%$ of all measurements being acceptable

\begin{tabular}{|l|c|c|}
\hline & Lower limit & Upper limit \\
\hline Synthetic samples & $-14 \%$ & $+11 \%$ \\
Natural samples & $-39 \%$ & $+17 \%$ \\
\hline
\end{tabular}

few available data points as if the variability of their results was greater.

In other words, the data sets were reasonably representative of actual water sources, but further studies have to focus specifically on the measurement of concentrations at or below say 0.5 $\mathrm{mg} / \mathrm{l}$, as this will be the critical region for water fluoridation plant operation.

\section{Does the uncertainty around fluoride measurement present an obstacle to water fluoridation?}

If nothing is done to improve measurement accuracy, or to demonstrate with a more comprehensive study that the problem is smaller than it now seems, then it would. In the example at the beginning of the paper, it was shown that undermeasurement of $30 \%$ would make it extremely difficult to stay within the allowed dosing tolerances. But the author has no doubt that the South African water industry, internationally known for decades for its drive and innovation, has adequate expertise and resources to successfully resolve this problem, provided its leadership shows the necessary will.

\section{Is fluoride measurement error the only significant source of error?}

The only systematic study of total fluoride dosing error known to the author has recently been published (Lalumandier et al., 2001). Operators at 1280 fluoridation plants in 12 US states responded, amongst others, to a question of how close they could maintain the fluoride dosing level to the optimal level. This effectively means that operators had to assess themselves without any external controls, which would probably lead to optimistic estimates. The results, briefly summarised, were:

- A total of $25.9 \%$ responded that they could maintain the concentration within $0.1 \mathrm{mg} / \mathrm{l}$ of the optimal level; $49.3 \%$ could maintain it between 0.1 and $0.2 \mathrm{mg} / \mathrm{l}$ from the optimal level and $19.5 \%$ could stay between 0.2 to $0.3 \mathrm{mg} / \mathrm{l}$ of the optimal level.

- For the large plants (above a capacity of about $4 \mathrm{Ml} / \mathrm{d}$ ) the performance was significantly better, with $33.5 \%$ of the large plants maintaining the dosage within $0.1 \mathrm{mg} / \mathrm{l}$ of the optimal, as opposed to $21.3 \%$ for the small plants.

- The two main causes for the dosing error were reported to be problems with feeding equipment (18.4\%) and variations in raw water flow (12.8\%).

- Poor training of operators was blamed for the dosing error in $8.7 \%$ of the responses.

These survey results are interpreted as follows:

- Most US treatment plants would not be able to stay within the tolerances allowed by the SA Regulations. 
- Measurement error (more difficult to detect) is not amongst the top two reasons for dosing error, indicating that the allowable measurement error should be set at a significantly lower level than the allowable total dosing error.

- Proper training of operators provides one way to improve dosing performance.

\section{Closure}

This paper presents more questions than answers. It is primarily intended to alert those water chemists and operators grappling with the pending implementation of water fluoridation in South Africa to the uncertainties surrounding the measurement of fluoride in water. It was shown that very good accuracy for fluoride measurement is a prerequisite for staying within the narrow dosing tolerances allowed by the SA Regulations.

Water providers are currently considering their technological options for expensive, sophisticated dosing and monitoring equipment for the pending fluoridation. Now is the time to undertake an urgent, systematic study of the fluoridation measurement options, pertaining to our typical source waters with their own unique potential for analytical interference. In order to get water fluoridation right, the SA water industry needs a set of practical, locally relevant guidelines for fluoride measurement, which can only be provided through the collaboration of the water chemists and process controllers in water treatment practice.

\section{Acknowledgments}

The CSIR (Dr. Carlsson and Ms. Elizabeth Truter of Watertek), the SABS (Mr. Chris Fouche and Ms. Marie McKay of the WATERCHECK programme) and MEDUNSA (Prof.JB du Plessis) generously shared the data reported on in this paper. Prof. Paul Coetzee of the Department of Chemistry at the Rand Afrikaans University provided better perspective on the analytical methods and their susceptability to interferences.

\section{References}

DEPARTMENT OF HEALTH (2000) Regulation No. 873 Regulations under the Health Act, 1977 (Act No. 63 of 1977) Regulations on Fluoridating Water Supplies. Government Gazette, 8 September 2000, Vol. 423, No. 21533, Pretoria.

LALUMANDIER JA, HERNANDEZ LC, LOCCI AB and REEVES TG (2001) US drinking water: Fluoridation knowledge level of water plant operators. J. Public Health Dent. 61 (2).

STANDARD METHODS (1985) Standard Methods for the Examination of Water and Wastewater (16 ${ }^{\text {th }}$ edn.) Published jointly by the American Public Health Association, the American Water Works Association and the Water Pollution Control Federation, Washington DC. 